\title{
Non-Surgical Management Of Large Periapical Lesion With Combination Of Calcium Hydroxide And Chlorhexidine Gel As Intracanal Medicament -A Case Report
}

\author{
${ }^{1}$ Dr.Abdu Semeer Palottil, ${ }^{2}$ Dr.Unnikrishna K, ${ }^{3}$ Dr.Ramesh Kumar M, MDS, \\ ${ }^{4}$ Dr.Jayasree S, MDS, MDS. \\ 1. Junior Resident, Dept of Endodontics Govt Dental College Calicut, Kerala, India \\ 2. Junior Resident, Dept of Endodontics Govt Dental College Calicut, Kerala, India \\ 3. Professor \& head, Dept of Endodontics Govt Dental College Calicut, Kerala, India \\ 4. Associate professor, Dept of Endodontics Govt Dental College Calicut, Kerala, India
}

\begin{abstract}
A patient reported to the department of conservative dentistry and endodontics with a large periapical lesion in relation to maxillary right lateral incisor and canine. Conservative nonsurgical root canal therapy was performed with calcium hydroxide in combination with chlorhexidine gel as intracanal medicament. Al most complete periapical healing was observed at the 6-month recall. This case report confirms that combination calcium hydroxide and chlorhexidine gel have synergistic effect in improving the antimicrobial properties that enhances periapical healing
\end{abstract}

Key Words: periapical, non surgical, calcium hydroxide

\section{Introduction}

Periapical lesion most often occur as a result of caries or trauma usually undetected unless it is presented as swelling or sinus tract or tooth migration. Based on histologic finding a periapical lesion can be a granuloma or a cyst. Aroud $85 \%$ of the periapical lesion is diagnosed as granuloma, $15 \%$ of cases are cysts. Earlier periapical lesions are managed by surgical intervention. Now with more understanding of biology of wound healing there is literature evidence that most of the periapical granuloma and pocket cyst heal by non surgical endodontic management. So the initial management of choice of this condition should be conservative non surgical endodontic treatment. If conservative non surgical endodontic treatment and retreatment fails, then only we have to go for more invasive surgical treatment option

\section{Case Report}

A 19 year old boy reported in department of conservative dentistry and endodontics Govt dental college Calicut with swelling in upper right region. Previous history revealed that there was a history of trauma that region one year before. Medical history was non contributory

Clinical examination revealed obliterated vestibular sulcus in upper right region in relation to tooth 11 , 12 , 13. Some amount of tooth migration has occurred. Maxillary right lateral and canine were slightly discolored Rdiographic examination revealed a periapial lesion of size $2 \mathrm{~cm}$ diameter in relation to tooth 12,13

Vitality checking was done with Roeko Endo-Frost (Coltene and Whaledent) showed no response with 12, 13.

Treatment plan decided was to undergo non surgical root canal treatment and if the lesion not responding to undergo surgical management. Treatment plan was explained to the patient and an informed consent was obtained. Access cavity was opened under rubber dam and wedgets, working length was determined using a combination of electronic apex locator (RAYPEX 6 VDW) and periapical radiographs. Root canal was enlarged to no.60k file using hypochlorite and chlorhexidine as irrigants. Root canal dried with paper points and calcium hydroxide mixed with chlorhexidine gel paste was placed as an intracanal medicament. After 7 days access cavity reopened, canal was irrigated with saline solution and obturated using gutta percha (sure-endo,korea) and zinc oxide sealer by lateral condensation technique. Patient was recalled at one, three, Six month intervals showed reduction in radiolucency and finally complete resolution of the lesion. 


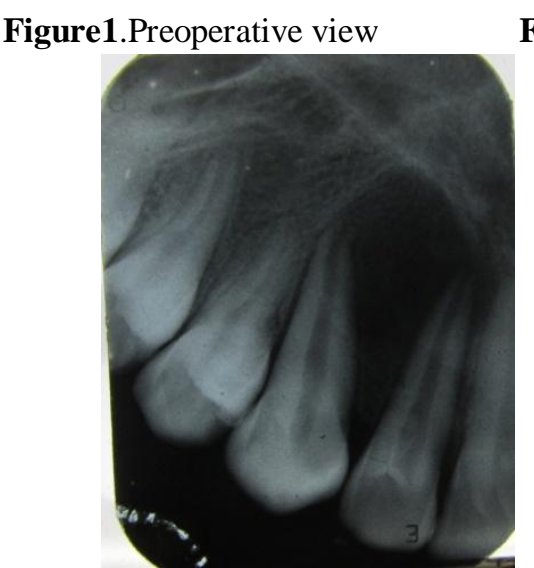

Figure4. 3 Month Review

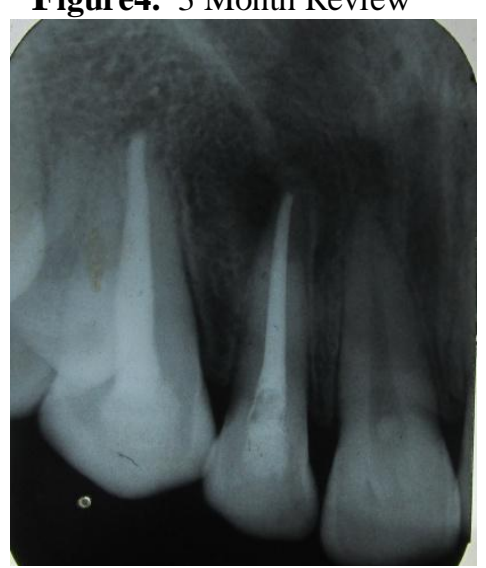

Figure3. 1 Month Review
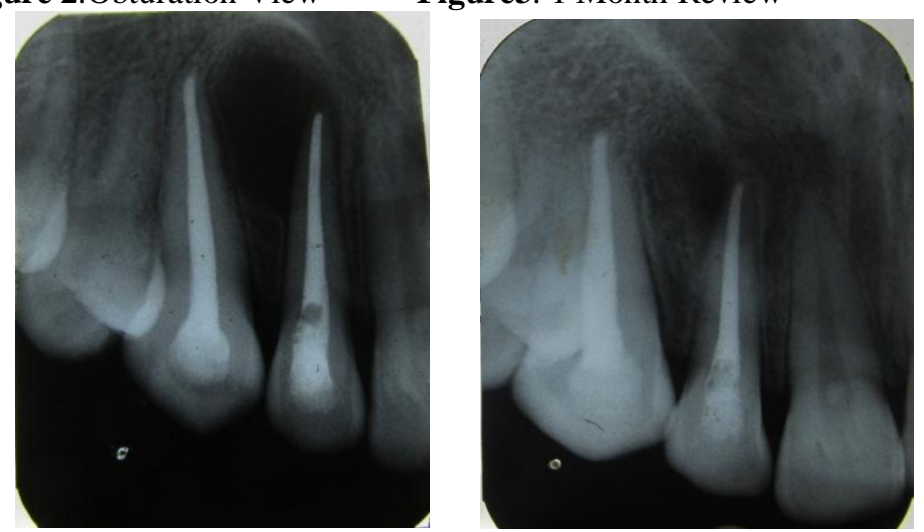

Figure5. 6 Month Review

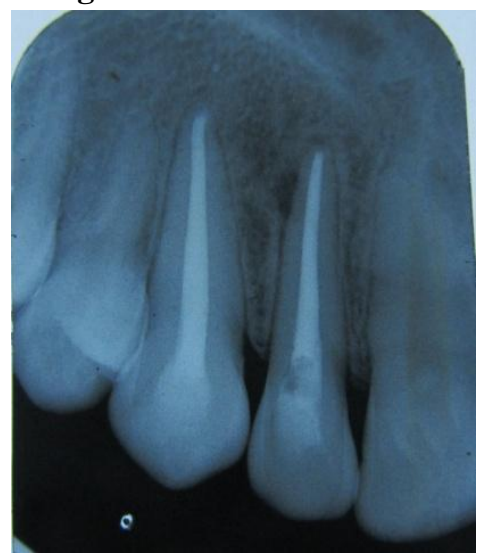

\section{Discussion}

Satisfactory wound healing is the ultimate goal in the management of tooth with large periapical lesion. Understanding of wound healing is as important as knowing the pathogenesis of disease. Presence of microbial organism is responsible for the development and progression of periapical lesion. So the success of the nonsurgical endodontic treatment is depending on appropriate cleaning, shaping, asepsis, and filling of the root canal. It has been said that proper sterilization and disinfection of the root canal and periradicular region results in good healing of periapical diseases in adults.

Calcium hydroxide is the most commonly used medicament for the asepsis of the root canal because of its high alkalinity and antibacterial activity. The antimicrobial effects of calcium hydroxide have been evaluated by clinical studies where calcium hydroxide has been shown to successfully disinfect root canals following 1 month dressing in $97 \%$ of treated cases. Calcium hydroxide plays an important role in endodontics due to its ability to induce hard tissue formation, its antibacterial effect and its ability to act as a physical barrier to prevent root canal reinfection. In an attempt to enhance the antimicrobial activity of $\mathrm{Ca}(\mathrm{OH})_{2}$, different substances have been used as vehicles. Researchers have studied the combination of calcium hydroxide $(\mathrm{Ca}(\mathrm{OH}) 2)$ and $\mathrm{CHX}$. Chlorhexidine gluconate has been widely used as an endodontic irrigant because of its antimicrobial activity against Gram-positive and Gram-negative microorganisms. (4) Chlorhexidine gluconate may also present residual antimicrobial activity on the dentin surface after prolonged contact (at least one week) with the root canal. Recent studies have suggested that $\mathrm{CHX}$ could be used in combination with $\mathrm{Ca}(\mathrm{OH})_{2}$ to improve antimicrobial efficacy against $\mathrm{Ca}(\mathrm{OH})_{2}-$ resistant microorganisms. Evans $\mathrm{MD}$ et al(2003) found that calcium hydroxide paste with $2 \%$ chlorhexidine was significantly more effective at killing E. faecalis in the dentinal tubules than calcium hydroxide with water.(1) Gomes et al (2006) conclued that $2 \% \mathrm{CHX}$ gel $+\mathrm{Ca}(\mathrm{OH}) 2$ showed better antimicrobial activity than $\mathrm{Ca}(\mathrm{OH}) 2$ manipulated with sterile water.(3) The present study healed almost completely with calcium hydroxide and chlorhexidine as intracanal medicament suggesting that combination of calcium hydroxide and chlorhexidine have synergistic fashion that enhances their efficacy.

\section{Conclusion}

The present case healed completely after non surgical rootcanal therapy. This confirms that even large periapical lesions can respond favorably to nonsurgical treatment if thourough chemomechanical preparation and intracanal medication were given. 


\section{Reference}

[1]. Evans MD, Baumgartner JC, Khemaleelakul SU, Xia T. Efficacy of calcium hydroxide: chlorhexidine paste as an intracanal medication in bovine dentin. J Endod 2003 May;29(5):338-9.

[2]. Silveira CF, Cunha RS, Fontana CE, et al. Assessment of the antibacterial activity of calcium hydroxide combined with chlorhexidine paste and other intracanal medications against bacterial pathogens. Eur J Dent 2011 Jan;5(1):1-7

[3]. Gomes BP, Vianna ME, Sena NT, Zaia AA, Ferraz CC, de Souza Filho FJ. In vitro evaluation of the antimicrobial activity of calcium hydroxide combined with chlorhexidine gel used as intracanal medicament. Oral Surg Oral Med Oral Pathol Oral Radiol Endod 2006 Oct;102(4):544-50 Epub 2006 Aug 4

[4]. Kanisavaran ZM. Chlorhexidine gluconate in endodontics: an update review. Int Dent J 2008 Oct;58(5):247-57.

[5]. Mohammadi Z, Abbott PV. The properties and applications of chlorhexidine in endodontics. Int Endod J 2009 Apr;42(4):288-302 doi: 101111/j1365-2591200801540x Epub 2009 Feb 7. 